\title{
Osteopontin deficiency protects mice from cholesterol gallstone formation by reducing expression of intestinal NPC1L1
}

\author{
JING LIN ${ }^{1}$, WEI-QING SHAO ${ }^{1}$, QING-ZHI CHEN ${ }^{2}$, WEN-WEI ZHU ${ }^{1}$, \\ LU LU $^{1}$, HU-LIANG JIA ${ }^{1}$ and JIN-HONG CHEN ${ }^{1}$ \\ ${ }^{1}$ Department of General Surgery, Huashan Hospital, Fudan University, Shanghai 200040; \\ ${ }^{2}$ Shanghai Huayu Private Middle School, Shanghai 200231, P.R. China
}

Received December 19, 2016; Accepted June 6, 2017

DOI: $10.3892 / \mathrm{mmr} .2017 .6774$

\begin{abstract}
Homeostasis of cholesterol is regulated by absorption in the intestine and synthesis in the liver. The authors previously demonstrated that OPN (osteopontin) exhibits the ability to alter hepatic cholesterol metabolism, thus affecting cholesterol gallstone formation in mice. The present study investigated the role of OPN in cholesterol gallstone formation, focusing on its effect on intestinal absorption of cholesterol. OPN gene knockout $\left(\mathrm{OPN}^{-/}\right)$mice and wild-type mice were respectively fed with a chow or lithogenic diet (LD) for 8 weeks. Following an 8-week LD period, the incidence of gallstone, bile composition, level of serum and fecal lipids and the expression of intestinal associated genes were analyzed. $\mathrm{OPN}^{-/}$mice were protected from gallstone formation induced by 8 weeks' LD-feeding. This protective effect from OPN deficiency was associated with alterations in bile composition, including a reduced concentration of biliary cholesterol. Additionally, plasma cholesterol level was decreased in $\mathrm{LD}$-fed $\mathrm{OPN}^{-/}$mice. The alterations primarily resulted from the decreased expression of intestinal Niemann-Pick C1-like (NPC1 L) 1, which is important in the intestinal absorption of cholesterol. The present study demonstrated that OPN deficiency reduced intestinal
\end{abstract}

Correspondence to: Professor Jin-Hong Chen, Department of General Surgery, Huashan Hospital, Fudan University, 12 Middle Urumqi Road, Shanghai 200040, P.R. China

E-mail: jinhongch@hotmail.com

Abbreviations: OPN, osteopontin; OPN ${ }^{-1}$, OPN gene knockout; WT, wild-type; CD, chow diet; LD, lithogenic diet; NPC1L1, Niemann-Pick C1-like L1; ABCG5/8, ATP-binding cassette, sub-family G, member 5/8; ACAT2, acetyl-Coenzyme A acetyltransferase 2; ASBT, apical sodium-dependent bile acid transporter; ILBP, ileal lipid binding protein; OST $\alpha / \beta$, organic solute transporter $\alpha / \beta ;$ ABCA1, ATP-binding cassette, sub-family A, member 1 ; LXR $\alpha$, liver X receptor $\alpha$; PPAR $\delta$, peroxisome proliferator activated receptor $\delta$; CSI, cholesterol saturation index; SD, standard deviation

Key words: cholesterol, gallstone formation, mice, Niemann-Pick C1-like L1, osteopontin absorption of cholesterol by suppressing the expression of NPC1L1, thus protecting mice from cholesterol gallstone formation.

\section{Introduction}

Gallstone disease is a global major health problem, and its attendant complications and comorbidities impose a huge financial burden on health care economy (1-4). Gallstone disease is a disease with multiple factors, mainly caused by the complex interaction of genetic factors and environmental factors (5). A prerequisite for cholesterol gallstone formation is biliary precipitation of excess cholesterol as solid crystals $(6,7)$. The most important source of biliary cholesterol is preformed plasma lipoprotein cholesterol which is mainly absorbed from the diet in small intestine, whereas only a minor contribution of cholesterol is generated by hepatic new synthesis or hydrolysis of cholesteryl ester stores (8). Dietary cholesterol is trafficked into enterocyte by Niemann-Pick C1-like 1 (NPC1L1) protein (9), and can be reversely transported to the intestinal lumen by ATP-binding cassette, sub-family G, member 5/8 (ABCG5/8) (10,11). After cholesterol passes through the enterocyte membrane, it should be esterified by acetyl-Coenzyme A acetyltransferase 2 (ACAT2); subsequent to this, it is transported in the chylomicrons circulating then reach the liver for biliary secretion $(12,13)$. Therefore, a high fat intake increases the risk of developing gallstone. And inhibiting NPC1L1 expression suppresses intestinal absorption of cholesterol, reduces plasma cholesterol level and prevents the development of gallstone (14).

Osteopontin (OPN) is a soluble cy tokine and a matrix-associated protein presenting in the majority of tissues and body fluids (15). Previous study found that plasma OPN level had a significant correlation with plasma cholesterol concentration in human (16). Our previous study demonstrated that OPN was involved in the pathogenesis of gallstone disease $(17,18)$, and that OPN altered hepatic cholesterol metabolism thus affecting cholesterol gallstone formation in mice (19). Because the homeostasis of cholesterol is regulated by both absorption in intestine and synthesis in liver $(20,21)$, it is also important to discover the role of OPN in intestinal cholesterol absorption. Therefore, we investigated the mechanism 
of OPN in cholesterol gallstone formation focusing on its effect on intestinal absorption of cholesterol in this study.

\section{Materials and methods}

Animals, diets and sample collection. WT mice were purchased from Fudan University (Shanghai, China). $\mathrm{OPN}^{-/ 2}$ mice in congenic background were purchased from Jackson Laboratory (Bar Harbor, ME, USA). Mice were housed at $22 \pm 2^{\circ} \mathrm{C}$ and $60 \pm 10 \%$ relative humidity in a specific pathogen-free environment, with a 12:12 h light: dark cycle. $\mathrm{WT}$ and $\mathrm{OPN}^{-/}$male mice between 8 and 10 weeks of age were fed a chow diet (CD) or LD (CD supplemented 15\% fat, $2 \%$ cholesterol, and $0.5 \%$ cholic acid) for up to 8 weeks. Bile was collected, and crystal analysis was immediately performed. Blood was collected via right ventricle heart puncture. Feces was collected from individually housed mice for $72 \mathrm{~h}$. The tissues were harvested and then snap-frozen in liquid nitrogen for protein and RNA isolation or fixed in 4\% paraformaldehyde overnight for histological analysis. All animals received humane care and all experimental procedures were conducted in conformance with principles of the National Institutes of Health guide for the care and use of laboratory animal (NIH Publications No. 8023) and approved by the Animal Ethics Committee of Fudan University.

Gallstone formation analysis and biochemical detections of bile, serum and feces. Gallstone were defined as macroscopically visible stones, whereas crystals were defined with polarizing light microscopy (Olympus, Tokyo, Japan). The fecal lipids and bile acids were extracted as previously described $(8,22)$. Cholesterol, bile acids and triglyceride levels were measured with assay kits from KHB (Shanghai, China), and phospholipid content was measured using an assay kit from Wako (Osaka, Japan) according to the manufacturers' instructions. All the biochemical measures were assayed in triplicate 3 times. Then the cholesterol saturation index (CSI) was calculated according to Carey's critical tables (23).

Quantitative real-time PCR analysis. Total RNAs were collected from the proximal segment of small intestine which was equally randomly divided into 5 segments or liver in each mouse with the RNAprep Pure Tissue kit (TianGen, Beijing, China) according to the manufacturer's instructions. Random primers (Takara, Shiga, Japan) were used for reverse transcription of total RNA to complementary DNA. Quantitative real-time PCR was performed using SYBR-Green I chemistry (TianGen) and the ABI 7900HT Fast Real-Time PCR System (Applied Biosystem, Shanghai, China). The gene-specific primer sequences are shown in Table I. Messenger RNA (mRNA) expression levels were calculated relative to the housekeeping gene GAPDH and further normalized to the expression levels of the respective controls following the basis of the relative expression method (24).

Western blot analysis. The entire proximal segment of small intestine that was randomly divided into 5 equal segments were lysed in ice-cold RIPA buffer supplemented with protease inhibitors. Protein concentration of the extracts was measured by the BCA Protein Assay kit (Thermo, Shanghai, China). The protein was analyzed by $8 \%$ SDS-PAGE and transferred to Poly (vinylidene fluoride) membranes. Anti-NPC1L1 antibody (Santa Cruz, Shanghai, China) was used at a 1:300 dilution. A 1:10,000 dilution of rabbit anti-goat immunoglobulin G-HRP (Santa Cruz) was used as a secondary antibody. After probing individual antibodies, the antigen-antibody complex was visualized by Enhanced Chemiluminescence Reagents Supersignal (TianGen, Beijing, China). The relative average protein level was determined by densitometry using Image $\mathbf{J}$ software (NIH, Maryland, and USA).

Histopathological and immunohistochemistry analysis. Tissues were fixed in $4 \%$ paraformaldehyde overnight and then transferred to $70 \%$ ethanol prior to routine processing and staining with hematoxylin and eosin. Formalin-fixed tissues were embedded in paraffin and cut into $3 \mathrm{~mm}$ sections. Sample slides were incubated with anti-NPC1L1 antibodies (Santa Cruz) with a 1:75 dilution, followed by $30 \mathrm{~min}$ incubation with an HRP-labeled polymer secondary antibody (Santa Cruz). Sections were viewed with a Nikon ECLIPSE E600 microscope (Nikon, Tokyo, Japan) using x10 objective lenses, and images were acquired with a SPOT INSIGHT ${ }^{\mathrm{TM}}$ digital color camera, model 3.2.0 (Sterling, Heights, MI). Quantification of immunoreactivity was performed on digitally captured color images saved as TIFF files and analyzed using Image-Pro plus 6.0 software (Media Cybernetics, Florida, USA). The blind histopathological and immunohistochemistry analysis were conducted by a pathologist of Fudan University.

Statistical analysis. Data are demonstrated as mean \pm standard deviation (SD) unless otherwise noted. The statistical significance of differences between the means of the experimental groups was evaluated with unpaired Student's $t$ test (GraphPad, La Jolla, CA, USA). A difference was considered to be statistically significant at $\mathrm{P}<0.05$.

\section{Results}

OPN deficiency reduced gallstone formation in LD-fed mice. Neither WT mice $(0 / 6)$ nor $\mathrm{OPN}^{-/-}$mice $(0 / 6)$ showed crystals or gallstones when fed a CD. When fed with LD for 8 weeks, all WT mice (6/6) developed gallstones, whereas the penetrance in $\mathrm{OPN}^{-/}$mice was $16.7 \%$ (1/6). Compared to those of $\mathrm{OPN}^{-/-}$mice, the gallbladder bile of WT mice appeared turbid and full of precipitates and stones. Microscopic examination of the gallbladder bile revealed cholesterol crystals in WT mice, whereas those of $\mathrm{OPN}^{-/-}$mice were largely free of cholesterol precipitates (Fig. 1).

OPN deficiency altered the cholesterol content and CSI in $L D$-fed mice. The histology examinations of gallbladder and ileum were similar in WT mice and $\mathrm{OPN}^{-/-}$mice (Fig. 2). And the body weight and gallbladder volume between two strains showed no difference (Table II). The cholesterol content of two genotypes mice were also similar when fed CD. After being fed with LD for 8 weeks, OPN ${ }^{-/}$mice exhibited reduced in biliary cholesterol content and serum cholesterol profile compared to WT mice. But the fecal cholesterol content showed no statistically difference in two LD-fed strains (Table II). There was no difference in serum phospholipid, triglyceride and bile acids 
Table I. Primer sequences for quantitative real-time PCR in mice.

\begin{tabular}{lll}
\hline Gene & \multicolumn{1}{c}{ Sense primer $\left(5^{\prime} \rightarrow 3^{\prime}\right)$} & \\
\hline NPC1L1 & CTCTGCCCTCTGCAATGCTC & Ansimer $\left(5^{\prime} \rightarrow 3^{\prime}\right)$ \\
ABCG5 & AGGGCCTCACATCAACAGAG & GAACAGGCTGCCGAGTCTT \\
ABCG8 & TGCCCACCTTCCACATGTC & GCTGACGCTGTAGGACACAT \\
ACAT2 & CTACAAGCAAGACCCAAGAG & ATGAAGCCGGCAGTAAGGTAGA \\
ABCA1 & CGTTTCCGGGAAGTGTCCTA & CATGTGGTAGATGGTTCGG \\
LXR $\alpha$ & GATAGGGTTGGAGTCAGCA & GCTAGAGATGACAAGGAGGATGGA \\
PPAR 8 & TCCATCGTCAACAAAGACGGG & GGAGCGCCTGTTACACTGTT \\
OST $\alpha$ & AGGCAGGACTCATATCAAACTTG & ACTTGGGCTCAATGATGTCAC \\
OST $\beta$ & AGATGCGGCTCCTTGGAATTA & TGAGGGCTATGTCCACTGGG \\
ASBT & GTCTGTCCCCCAAATGCAACT & TGGCTGCTTCTTTCGATTTCTG \\
ILBP & CTTCCAGGAGACGTGATTGAAA & CACCCCATAGAAAACATCACCA \\
HMGCS & GCCGTGAACTGGGTCGAA & CCTCCGAAGTCTGGTGATAGTTG \\
HMGCR & CTTGTGGAATGCCTTGTGATTG & GCATATATAGCAATGTCTCCTGCAA \\
SREBP2 & GCGTTCTGGAGACCATGGA & AGCCGAAGCAGCACATGAT \\
LDLR & TGGCTGTTCCCACATCTG & ACAAAGTTGCTCTGAAAACAAATCA \\
LRP & ACTATGGATGCCCCTAAAACTTG & CTCGTCAATATCTTCACACCTG \\
PCSK9 & ACCCTCATAGGCCTGGAGTT & GCAATCTCTTTCACCGTCACA \\
GAPDH & TGTGTCCGTCGTGGATCTGA & CTGTGATGACCTCTGGAGCA \\
\hline
\end{tabular}
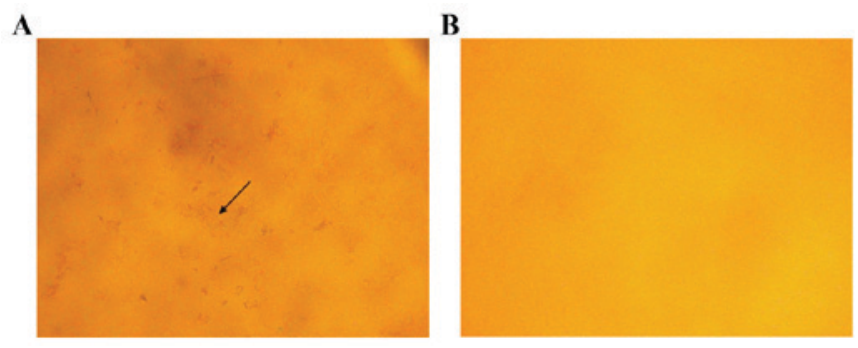

Figure 1. Loss of OPN protects against gallstone formation in LD-fed mice. Polarizing light microscopic examination of cholesterol crystals (the black arrows indicated) in representative bile from (A) WT and (B) $\mathrm{OPN}^{-/}$mice fed with LD for 8 weeks (original magnification, x10). WT, wild-type; OPN ${ }^{-/}$, OPN knockout; LD, lithogenic diet.

profiles between two LD-fed genotypes (Table III). And the fecal bile acids content showed no statistically difference in two strains. However, the LD-fed $\mathrm{OPN}^{-/-}$mice increased in biliary bile acids and biliary phospholipid content compared to LD-fed WT mice (Table III). This combined effect of biliary biochemical alterations led to a decreased CSI in LD-fed $\mathrm{OPN}^{-/}$mice (Table II), providing a biochemical mechanism for the phenotype that protecting $\mathrm{OPN}^{-/}$mice from cholesterol gallstone formation.

OPN deficiency suppressed the expression of intestinal NPC1L1 in LD-fed mice. To understand the mechanisms responsible for the changes in biochemical contents, we profiled the expression of intestinal related genes. There was no significant difference in genes expression between the two genotypes on CD (Fig. 3A and B). When challenged with the $\mathrm{LD}$ for 8 weeks, $\mathrm{OPN}^{-/-}$mice showed no difference in the expression of apical sodium-dependent bile acid transporter (ASBT), ileal lipid binding protein (ILBP) and organic solute
$\mathbf{A}$
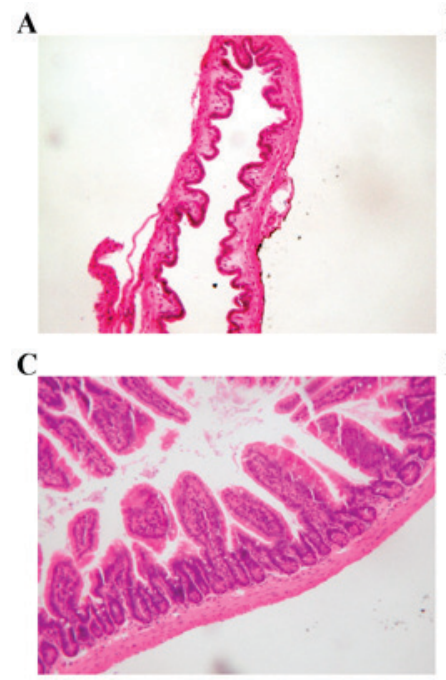

B

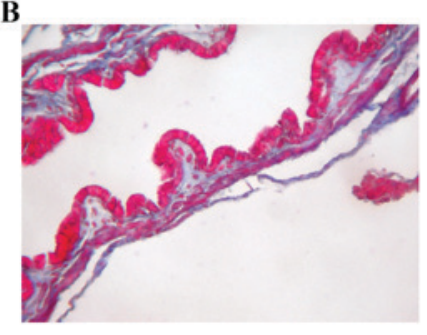

D

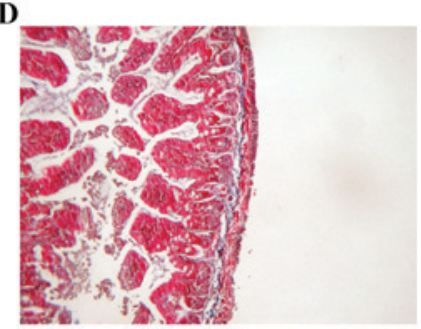

Figure 2. Histology examinations of gallbladder and ileum in WT and $\mathrm{OPN}^{-/-}$mice. Histology examinations of gallbladder from (A) WT and (B) $\mathrm{OPN}^{-/}$mice fed with LD for 8 weeks (original magnification, x200). Histology examinations of ileum from (C) WT and (D) $\mathrm{OPN}^{-/}$mice fed with LD for 8 weeks (original magnification, x200). WT, wild-type; $\mathrm{OPN}^{-/}$, OPN knockout; LD, lithogenic diet.

transporters $\alpha / \beta(\mathrm{OST} \alpha / \beta)$, which are involved in intestinal reabsorption of bile acids (Fig. 3C). Among the intestinal cholesterol transporters, the expression of NPC1L1 had an almost $50 \%$ reduction in LD-fed $\mathrm{OPN}^{-/-}$mice, whereas the expression of ABCG5/8, ATP-binding cassette, sub-family A, member 1 (ABCA1), and ACAT2 was not affected (Fig. 3D). Then we detected the gene expression of liver $\mathrm{X}$ receptor $\alpha$ $(\mathrm{LXR} \alpha)$ and peroxisome proliferator activated receptor $\delta$ (PPARS), and found it was similar between two mice strains (Fig. 3D). Then we challenged the protein expression of 
Table II. The body weight, gallbladder volume and cholesterol content in WT and $\mathrm{OPN}^{-/-}$mice.

\begin{tabular}{|c|c|c|c|c|}
\hline \multirow[b]{2}{*}{ Parameters } & \multicolumn{2}{|c|}{$\mathrm{CD}$} & \multicolumn{2}{|c|}{ LD for 8 weeks } \\
\hline & WT & $\mathrm{OPN}^{-/-}$ & WT & $\mathrm{OPN}^{-/-}$ \\
\hline Body weight (g) & $20.1 \pm 1.06$ & $20.5 \pm 1.06$ & $20.3 \pm 0.75$ & $22.8 \pm 2.38$ \\
\hline Gallbladder volume $(\mu 1)$ & $23.2 \pm 7.57$ & $25.6 \pm 11.5$ & $27.7 \pm 9.14$ & $23.5 \pm 6.51$ \\
\hline Biliary cholesterol $(\mathrm{mmol} / \mathrm{l})$ & $2.58 \pm 0.67$ & $2.88 \pm 0.59$ & $8.81 \pm 1.14$ & $5.34 \pm 0.90^{\mathrm{a}}$ \\
\hline Serum cholesterol $(\mathrm{mmol} / \mathrm{l})$ & $1.85 \pm 0.15$ & $1.96 \pm 0.33$ & $3.10 \pm 0.56$ & $2.31 \pm 0.25^{\mathrm{a}}$ \\
\hline Fecal cholesterol output (mg/g feces/day) & $3.83 \pm 0.84$ & $3.77 \pm 0.73$ & $15.29 \pm 1.22$ & $17.08 \pm 2.11$ \\
\hline CSI & $0.48 \pm 0.12$ & $0.49 \pm 0.05$ & $1.19 \pm 0.13$ & $0.59 \pm 0.07^{\mathrm{b}}$ \\
\hline
\end{tabular}

The data are expressed as mean $\pm \mathrm{SD}$ ( $\mathrm{n}=6$ per groups). Statistical analysis was performed by unpaired Student's $\mathrm{t}$ test, ${ }^{\mathrm{a}} \mathrm{P}<0.05$, ${ }^{\mathrm{b}} \mathrm{P}<0.01$. WT, wild-type; $\mathrm{OPN}^{--}$, OPN knockout; $\mathrm{CD}$, chow diet; LD, lithogenic diet; CSI, cholesterol saturation index.

Table III. Lipids and bile acids profiles in $\mathrm{WT}$ and $\mathrm{OPN}^{-/-}$mice.

\begin{tabular}{|c|c|c|c|c|}
\hline \multirow[b]{2}{*}{ Parameters } & \multicolumn{2}{|c|}{$\mathrm{CD}$} & \multicolumn{2}{|c|}{ LD for 8 weeks } \\
\hline & WT & $\mathrm{OPN}^{-/-}$ & WT & $\mathrm{OPN}^{-/-}$ \\
\hline Biliary bile acids (mmol/l) & $56.65 \pm 6.08$ & $63.59 \pm 7.66$ & $79.79 \pm 11.0$ & $97.34 \pm 15.8^{\mathrm{a}}$ \\
\hline Biliary phospholipid (mmol/l) & $20.53 \pm 1.55$ & $21.99 \pm 2.82$ & $24.68 \pm 2.45$ & $30.48 \pm 4.80^{\mathrm{a}}$ \\
\hline Serum phospholipid (mg/dl) & $157.4 \pm 18.1$ & $167.0 \pm 31.0$ & $182.6 \pm 61.0$ & $190.7 \pm 22.5$ \\
\hline Serum bile acids $(\mu \mathrm{mol} / \mathrm{l})$ & $4.30 \pm 5.15$ & $4.17 \pm 3.13$ & $10.3 \pm 8.03$ & $13.5 \pm 5.25$ \\
\hline Serum triglyceride (mmol/l) & $0.32 \pm 0.19$ & $0.39 \pm 0.18$ & $0.46 \pm 0.73$ & $0.30 \pm 0.03$ \\
\hline Fecal bile acids output $(\mu \mathrm{mol} / \mathrm{g}$ feces $/$ day $)$ & $2.82 \pm 0.25$ & $3.17 \pm 0.75$ & $66.80 \pm 5.21$ & $71.3 \pm 11.80$ \\
\hline
\end{tabular}

The data are expressed as mean $\pm \mathrm{SD}$ ( $\mathrm{n}=6$ per groups). Statistical analysis was performed by unpaired Student's $\mathrm{t}$ test, ${ }^{\mathrm{a}} \mathrm{P}<0.05$, ${ }^{\mathrm{b}} \mathrm{P}<0.01$. WT, wild-type; OPN ${ }^{--}$, OPN knockout; $\mathrm{CD}$, chow diet; LD, lithogenic diet.

A

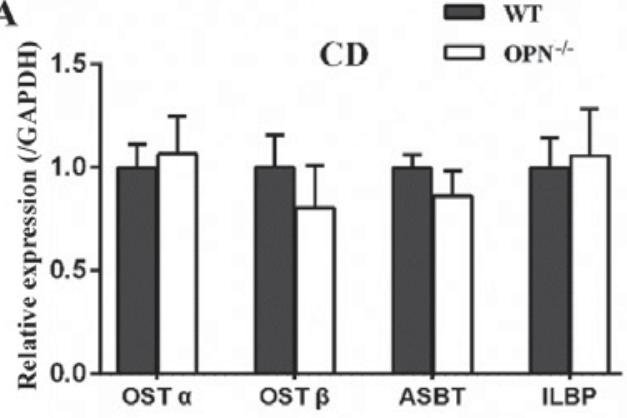

$c$

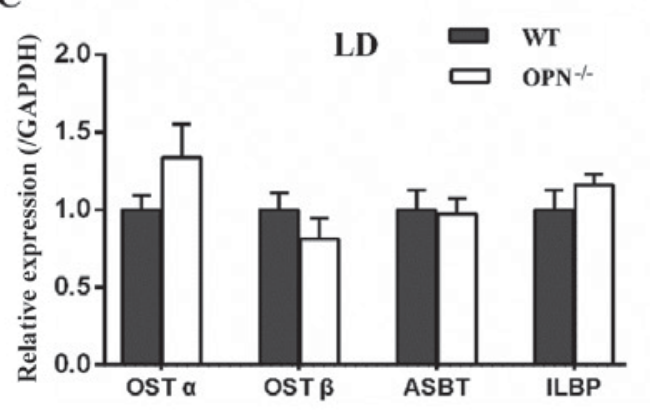

B

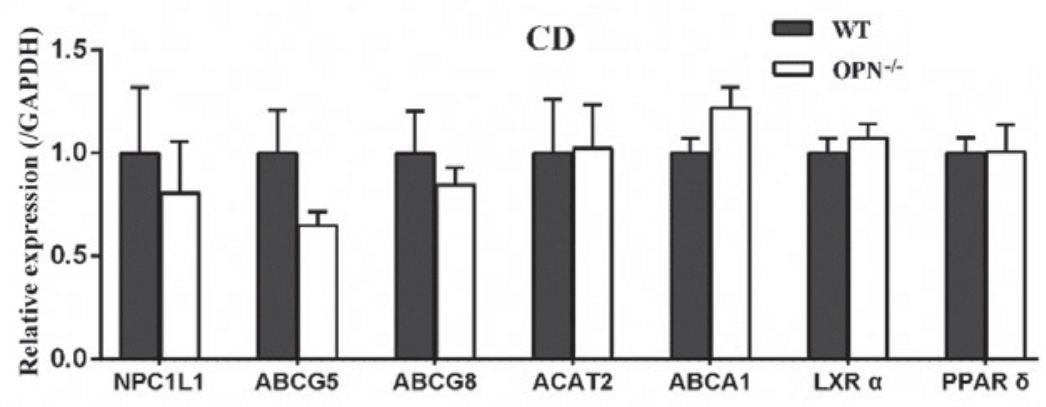

D

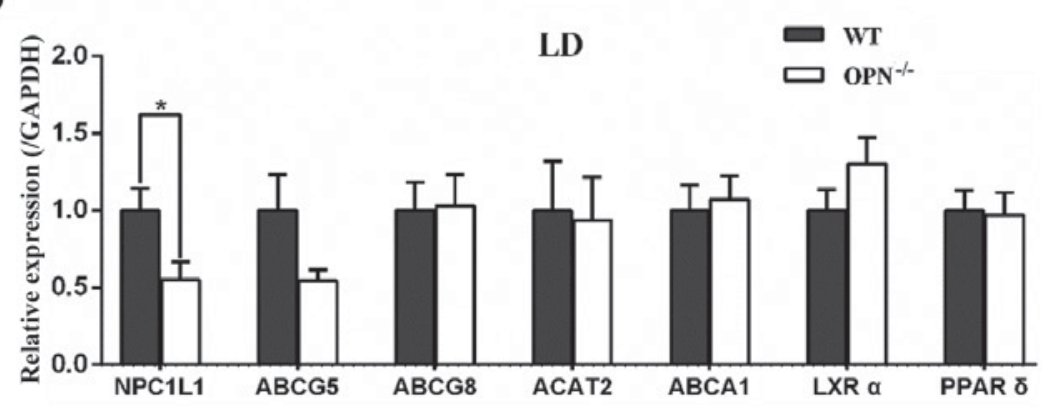

Figure 3. The mRNA expression of intestinal related genes in WT and $\mathrm{OPN}^{-/-}$mice. Quantitative real-time PCR analysis of mRNA levels for intestinal related genes from WT mice and $\mathrm{OPN}^{-/}$mice fed a (A and B) CD or (C and D) LD for 8 weeks. The data are expressed as the mean \pm SD ( $=6$ per group). Statistical analysis was performed by unpaired Student's t test, ${ }^{\mathrm{P}}<0.05$. WT, wild-type; OPN ${ }^{--}$, OPN knockout; CD, chow diet; LD, lithogenic diet. 
A

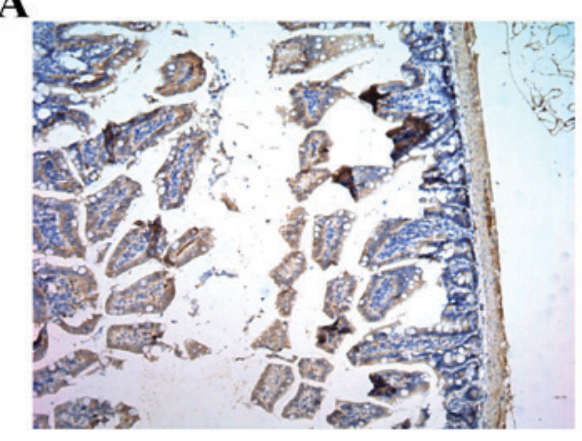

C

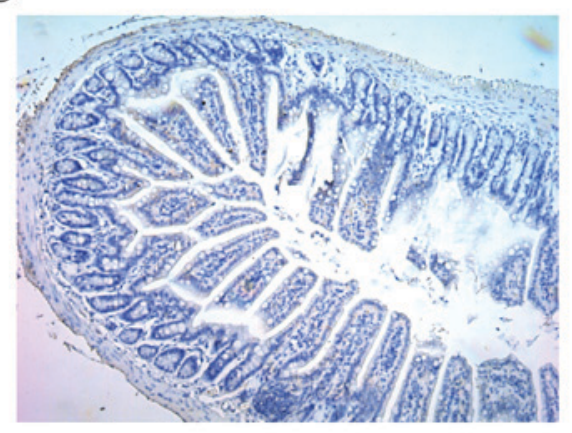

B

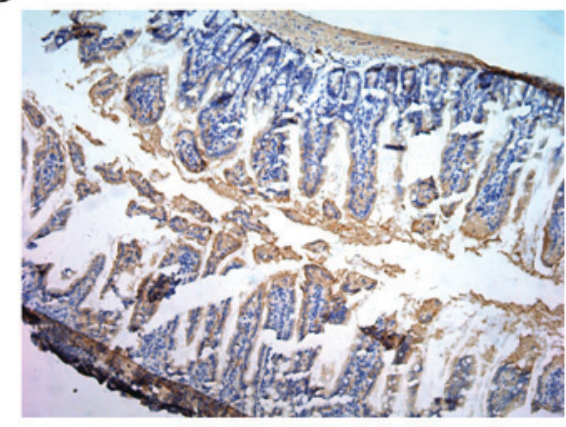

D

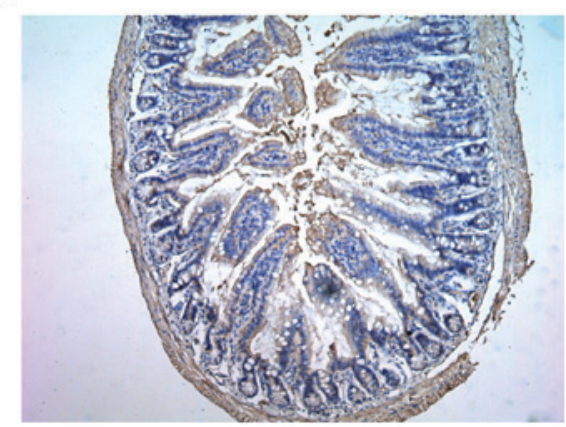

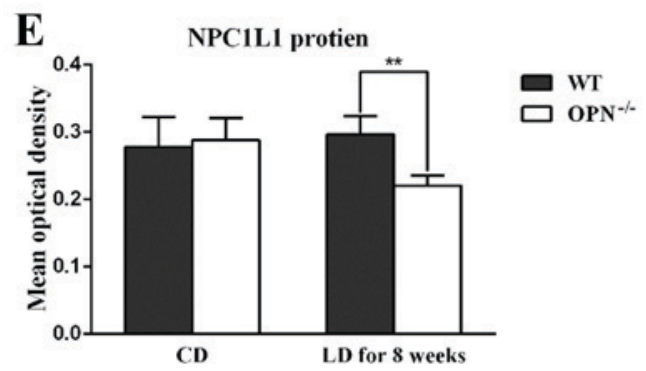

Figure 4. Immunohistochemistry analysis of NPC1L1 protein expression in mice. Ileum specimens from (A) CD-fed OPN ${ }^{-/}$, (B) CD-fed WT, (C) LD-fed $\mathrm{OPN}^{-/-}$, (D) LD-fed WT mice that were immunohistochemically stained for NPC1L1. (E) Quantitative immunohistochemical analysis of ileum NPC1L1 levels by mean optical density in mice. The data are expressed as the mean \pm SD ( $n=6$ per group). Statistical analysis was performed using unpaired Student's test, ${ }^{* *} \mathrm{P}<0.01$. WT, wild-type; OPN ${ }^{-/-}$, OPN knockout; CD, chow diet; LD, lithogenic diet.

intestinal NPC1L1 with immunohistochemistry, and found that the protein expression of NPC1L1 was similar in two CD-fed strains but decreased in LD-fed $\mathrm{OPN}^{-/-}$mice (Fig. 4). And the changed expression of NPC1L1 protein was also confirmed by western blot analysis (Fig. 5).

Because the homeostasis of cholesterol is regulated by both absorption in intestine and synthesis in liver, we also measured the expression of hepatic related genes. There were no significant differences in those genes between the two genotypes fed a CD (Fig. 6A). When challenged with the LD for 8 weeks the expression of proprotein convertase subtilisin/kexin type 9 (PCSK9) tended to be decreased in $\mathrm{OPN}^{-/-}$mice, but the change was not statistically significant. The expression of other hepatic related genes also showed no difference between two mice strains fed a LD (Fig. 6B).

\section{Discussion}

In this study, we found that OPN deficiency could attenuate the absorption of cholesterol by reducing intestinal expression of NPC1L1, thus protecting mice from diet-induced cholesterol gallstone formation.
$\mathrm{OPN}^{-/-}$mice was less susceptible to diet-induced gallstone than WT mice, which is consistent with our previous work (19). The body weight and gallbladder volume did not differ between the $\mathrm{OPN}^{-/-}$mice and WT mice fed with $\mathrm{CD}$ nor LD for 8 weeks. In addition, gallbladder histology was also similar in two strains. These findings indicate that the abnormalities of gallbladder are unlikely to be primarily responsible for the protection of LD-fed $\mathrm{OPN}^{-/}$mice from gallstone formation. As the prerequisite for cholesterol gallstone formation is the precipitation of excess cholesterol in bile as solid crystals, this protection of LD-fed $\mathrm{OPN}^{-/}$mice from gallstone formation may be due to the remarkably lower biliary cholesterol concentration, with greater levels of bile acids and phospholipid and consequently a lower CSI.

Then we observed that the content of serum cholesterol in $\mathrm{LD}^{-f e d ~} \mathrm{OPN}^{-/}$mice was lower than that of LD-fed WT mice. And the fecal cholesterol output in LD-fed $\mathrm{OPN}^{-/}$was increased in value, although the difference is not statistically significant. Additionally, the mRNA and protein expressions of NPC1L1, which is localized to the brush-border membrane of enterocytes $+(25)$ and plays an important role in intestinal cholesterol absorption (9), were both reduced in LD-fed 
A

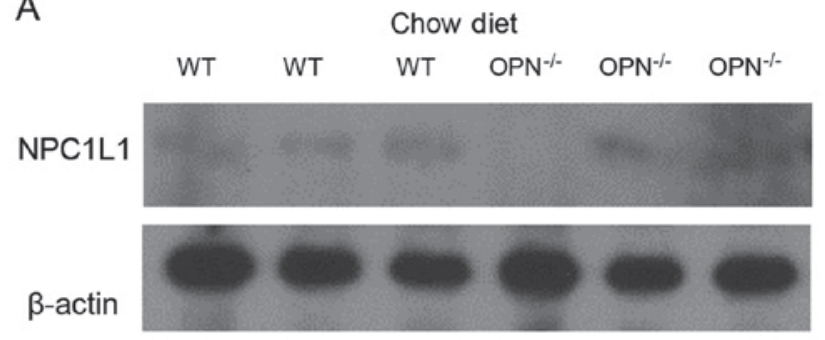

B

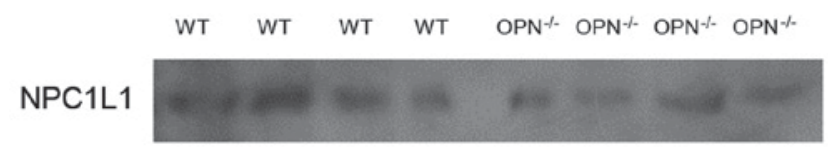

$\beta$-actin
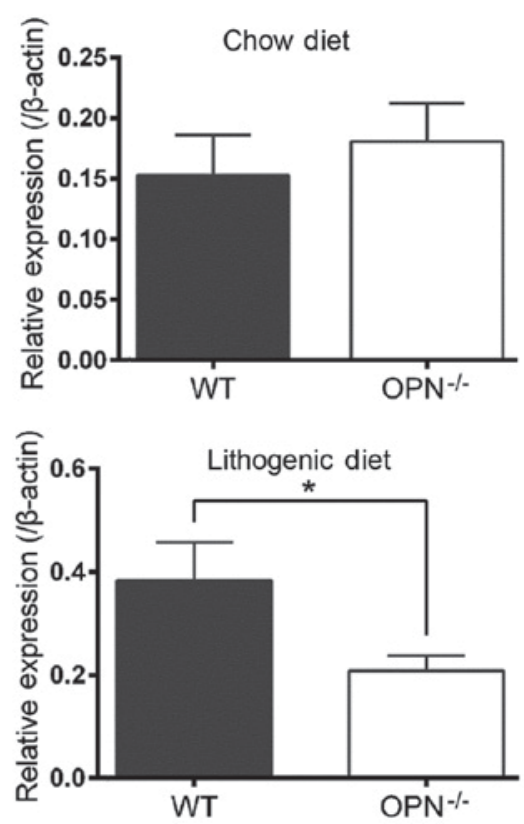

Figure 5. Western blot analysis of expression of protein NPC1L1 in mice. Ileum proteins were isolated from WT and OPN ${ }^{-/}$mice fed a (A) CD and (B) LD for 8 weeks, then analyzed by western blotting for NPC11 protein expression. The $\beta$-actin was selected as a control for gel loading. The relative average protein level was determined by densitometry. The data are expressed as the mean $\pm \mathrm{SD}$. Statistical analysis was performed using unpaired Student's $t$ test, "P<0.05. WT, wild-type; OPN ${ }^{-/}$, OPN knockout; CD, chow diet; LD, lithogenic diet.
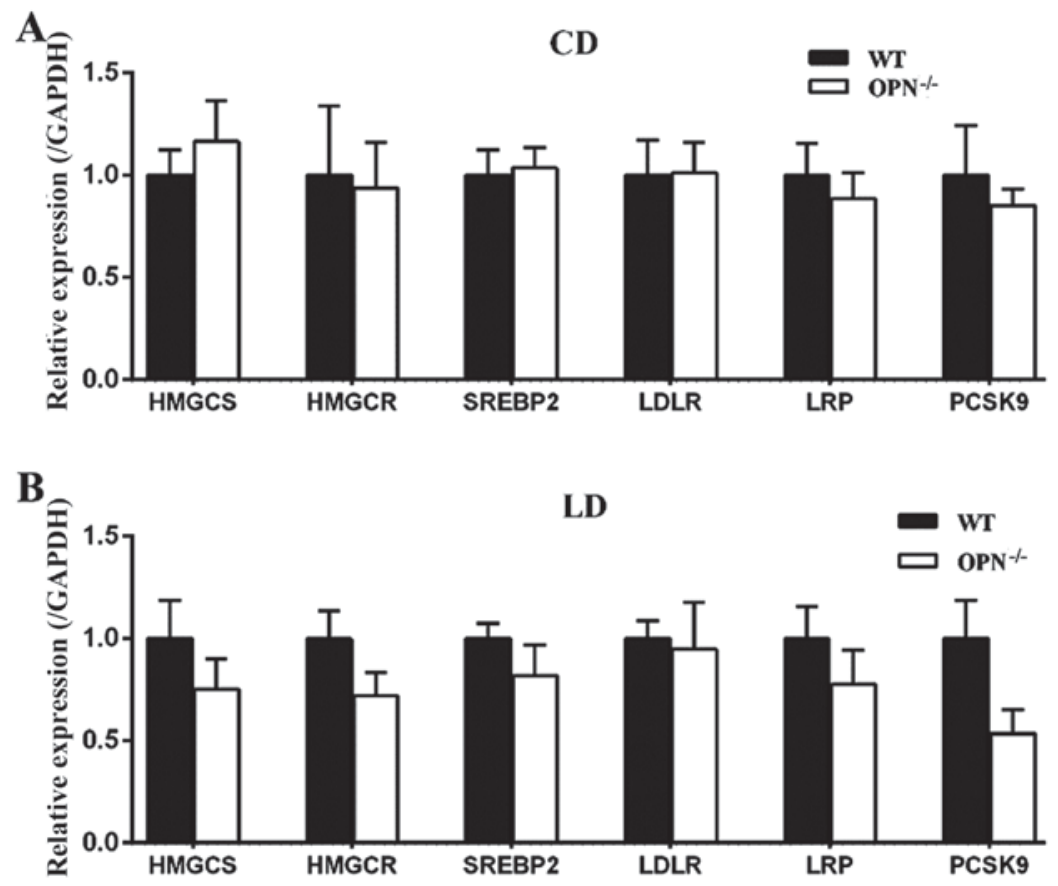

Figure 6. The mRNA expression of hepatic related genes in WT and $\mathrm{OPN}^{-/}$mice. Quantitative real-time PCR analysis of mRNA levels for hepatic related genes from WT mice and OPN ${ }^{-/-}$mice fed a (A) CD and (B) LD for 8 weeks. The data are expressed as the mean \pm SD (n=6 per group). Statistical analysis was performed by unpaired Student's t test. WT, wild-type; OPN ${ }^{-/}$, OPN knockout; CD, chow diet; LD, lithogenic diet.

$\mathrm{OPN}^{-/-}$mice. It is known that cholesterol derived from the intestine provides the first major source for cholesterol pool and influences biliary cholesterol secretion (26-28), and that high absorption of cholesterol enhances gallstone formation through this pathway (29). Therefore the participation of the NPC1L1 protein is very important, for it can determine the amount of cholesterol circulating to the liver and allow it to be excreted into bile. The increased level of intestinal NPC1L1 protein in gallbladder gallstone patients produces a high absorption of cholesterol (30). But inhibiting intestinal NPC1L1 expression reduces the intestinal absorption of cholesterol and prevents the formation of gallstone (31-34). Ezetimibe, which is a drug that blocks the expression of NPC1L1, could prevent cholesterol gallstone formation by effectively reducing intestinal cholesterol absorption and biliary cholesterol secretion $(34,35)$. Although we did not measure the cholesterol 
absorption rates during the mice experiments, these findings suggest that the protection of LD-fed $\mathrm{OPN}^{-/}$mice from gallstone formation may be partially resulted from the reduced intestinal absorption of cholesterol, which is caused by the decreased expression of intestinal NPC1L1. However, it is a limitation that we have not performed NPC1L1 overexpression assays in mice to confirm the function of NPC1L1.

The expression of intestinal NPC1L1 is mainly regulated by LXR $\alpha$ (36) and PPAR $\delta$ (37). Unfortunately, we found no difference in the expression of LXR $\alpha$ nor PPAR $\delta$ between two mice strains. It suggests that the effect of OPN deficiency on intestinal NPC1L1 expression may not function through the LXR $\alpha$ or PPAR $\delta$ pathway. Further studies are necessary in order to fully address the mechanism by which OPN deficiency leads to intestinal NPC1L1 suppression.

For the increased biliary bile acids content in LD-fed $\mathrm{OPN}^{-/}$mice, we did not find the expression of intestinal genes involved in reabsorption of bile acids differ between two strains. The reason might be that OPN could influence the expression of the cytochrome P450, family 7 , subfamily a, polypeptide 1 , the rate limiting enzyme of bile acids synthesis, as we found in our previous study (19).

In conclusion, we have described a previously unknown function of OPN, that it can affect intestinal absorption of cholesterol through NPC1L1, thus impacting the formation of gallstone in mice.

\section{Acknowledgements}

The present study was supported by the National Science Foundation of China (no. 81270536).

\section{References}

1. Sandler RS, Everhart JE, Donowitz M, Adams E, Cronin K, Goodman C, Gemmen E, Shah S, Avdic A and Rubin R: The burden of selected digestive diseases in the United States. Gastroenterology 122: 1500-1511, 2002.

2. Everhart JE and Ruhl CE: Burden of digestive diseases in the United States Part III: Liver, biliary tract, and pancreas. Gastroenterology 136: 1134-1144, 2009.

3. Kaechele V, Wabitsch M, Thiere D, Kessler AL, Haenle MM, Mayer $\mathrm{H}$ and Kratzer W: Prevalence of gallbladder stone disease in obese children and adolescents: Influence of the degree of obesity, sex, and pubertal development. J Pediatr Gastroenterol Nutr 42: 66-70, 2006.

4. Shaffer EA: Gallstone disease: Epidemiology of gallbladder stone disease. Best Pract Res Clin Gastroenterol 20: 981-996, 2006.

5. Portincasa P, Moschetta A and Palasciano G: Cholesterol gallstone disease. Lancet 368: 230-239, 2006.

6. Hofmann AF, Amelsberg A and VanSonnenberg E: Pathogenesis and treatment of gallstones. N Engl J Med 328: 1854-1855, 1993

7. Lamont JT and Carey MC: Cholesterol gallstone formation. 2. Pathobiology and pathomechanics. Prog Liver Dis 10: 165-191, 1992.

8. Turley SD, Daggy BP and Dietschy JM: Effect of feeding psyllium and cholestyramine in combination on low density lipoprotein metabolism and fecal bile acid excretion in hamsters with dietary-induced hypercholesterolemia. J Cardiovasc Pharmacol 27: 71-79, 1996.

9. Altmann SW, Davis HJ Jr, Zhu LJ, Yao X, Hoos LM, Tetzloff G, Iyer SP, Maguire M, Golovko A, Zeng M, et al: Niemann-Pick C1 like 1 protein is critical for intestinal cholesterol absorption. Science 303: 1201-1204, 2004.

10. Graf GA, Yu L, Li WP, Gerard R, Tuma PL, Cohen JC and Hobbs HH: ABCG5 and ABCG8 are obligate heterodimers for protein trafficking and biliary cholesterol excretion. J Biol Chem 278: 48275-48282, 2003.
11. Yu L, Li-Hawkins J, Hammer RE, Berge KE, Horton JD, Cohen JC and Hobbs HH: Overexpression of ABCG5 and ABCG 8 promotes biliary cholesterol secretion and reduces fractional absorption of dietary cholesterol. J Clin Invest 110: 671-680, 2002.

12. Grenier E, Garofalo C, Delvin E and Levy E: Modulatory role of PYY in transport and metabolism of cholesterol in intestinal epithelial cells. PLoS One 7: e40992, 2012.

13. Anderson RA, Joyce C, Davis M, Reagan JW, Clark M, Shelness GS and Rudel LL: Identification of a form of acyl-CoA: Cholesterol acyltransferase specific to liver and intestine in nonhuman primates. J Biol Chem 273: 26747-26754, 1998.

14. Tang W, Jia L, Ma Y, Xie P, Haywood J, Dawson PA, Li J and Yu L: Ezetimibe restores biliary cholesterol excretion in mice expressing Niemann-Pick C1-like 1 only in liver. Biochim Biophys Acta 1811: 549-555, 2011.

15. Wang KX and Denhardt DT: Osteopontin: Role in immune regulation and stress responses. Cytokine Growth Factor Rev 19: $333-345,2008$

16. Takemoto M, Tada K, Nakatsuka K, Moriyama Y, Kazui H, Yokote K, Matsumoto T, Saito Y and Mori S: Effects of aging and hyperlipidemia on plasma osteopontin level. Nihon Ronen Igakkai Zasshi 36: 799-802, 1999 (In Japanese).

17. Yang L, Chen JH, Cai D, Wang LY and Zha XL: Osteopontin and integrin are involved in cholesterol gallstone formation. Med Sci Monit 18: BR16-BR23, 2012.

18. Yang L, Chen JH, Cai D, Wang LY and Zha XL: Osteopontin plays an anti-nucleation role in cholesterol gallstone formation. Hepatol Res 41: 437-445, 2011.

19. Lin J, Shao WQ, Chen ZY, Zhu WW, Lu L, Cai D, Qin LX, Jia HL, Lu M and Chen JH: Osteopontin deficiency alters biliary homeostasis and protects against gallstone formation. Sci Rep 6: 30215, 2016.

20. Dietschy JM and Siperstein MD: Effect of cholesterol feeding and fasting on sterol synthesis in seventeen tissues of the rat. J Lipid Res 8: 97-104, 1967.

21. Spady DK and Dietschy JM: Sterol synthesis in vivo in 18 tissues of the squirrel monkey, guinea pig, rabbit, hamster, and rat. J Lipid Res 24: 303-315, 1983.

22. Nervi F, Marinović I, Rigotti A and Ulloa N: Regulation of biliary cholesterol secretion. Functional relationship between the canalicular and sinusoidal cholesterol secretory pathways in the rat. J Clin Invest 82: 1818-1825, 1988.

23. Carey MC: Critical tables for calculating the cholesterol saturation of native bile. J Lipid Res 19: 945-955, 1978.

24. Livak KJ and Schmittgen TD: Analysis of relative gene expression data using real-time quantitative PCR and the 2(-Delta Delta C(T)) Method. Methods 25: 402-408, 2001.

25. Davis HJ Jr, Zhu LJ, Hoos LM, Tetzloff G, Maguire M, Liu J, Yao X, Iyer SP, Lam MH, Lund EG, et al: Niemann-Pick C1 like 1 (NPC1L1) is the intestinal phytosterol and cholesterol transporter and a key modulator of whole-body cholesterol homeostasis. J Biol Chem 279: 33586-33592, 2004.

26. Buhman KK, Accad M,Novak S, Choi RS, Wong JS, Hamilton RL, Turley S and Farese RV Jr: Resistance to diet-induced hypercholesterolemia and gallstone formation in ACAT2-deficient mice. Nat Med 6: 1341-1347, 2000.

27. Wang HH and Wang DQ: Reduced susceptibility to cholesterol gallstone formation in mice that do not produce apolipoprotein B48 in the intestine. Hepatology 42: 894-904, 2005.

28. Amigo L, Quinones V, Mardones P, Zanlungo S, Miquel JF, Nervi F and Rigotti A: Impaired biliary cholesterol secretion and decreased gallstone formation in apolipoprotein E-deficient mice fed a high-cholesterol diet. Gastroenterology 118: 772-779, 2000.

29. Wang DQ, Zhang L and Wang HH: High cholesterol absorption efficiency and rapid biliary secretion of chylomicron remnant cholesterol enhance cholelithogenesis in gallstone-susceptible mice. Biochim Biophys Acta 1733: 90-99, 2005.

30. Jiang ZY, Jiang CY, Wang L, Wang JC, Zhang SD, Einarsson C, Eriksson M, Han TQ, Parini P and Eggertsen G: Increased NPC1L1 and ACAT2 expression in the jejunal mucosa from Chinese gallstone patients. Biochem Biophys Res Commun 379: 49-54, 2009.

31. Li Y, Li M, Wu S and Tian Y: Combination of curcumin and piperine prevents formation of gallstones in C57BL6 mice fed on lithogenic diet: Whether NPC1L1/SREBP2 participates in this process? Lipids Health Dis 14: 100, 2015.

32. Betters JL and Yu L: NPC1L1 and cholesterol transport. Febs Lett 584: 2740-2747, 2010. 
33. Valasek MA, Repa JJ, Quan G, Dietschy JM and Turley SD: Inhibiting intestinal NPC1L1 activity prevents diet-induced increase in biliary cholesterol in Golden Syrian hamsters. Am J Physiol Gastrointest Liver Physiol 295: G813-G822, 2008.

34. Wang HH, Portincasa P, Mendez-Sanchez N, Uribe $M$ and Wang DQ: Effect of ezetimibe on the prevention and dissolution of cholesterol gallstones. Gastroenterology 134: 2101-2110, 2008.

35. Zúñiga S, Molina H, Azocar L, Amigo L, Nervi F, Pimentel F, Jarufe N, Arrese M, Lammert F and Miquel JF: Ezetimibe prevents cholesterol gallstone formation in mice. Liver Int 28 935-947, 2008
36. Duval C, Touche V, Tailleux A, Fruchart JC, Fievet C, Clavey V, Staels B and Lestavel S: Niemann-Pick C1 like 1 gene expression is down-regulated by LXR activators in the intestine. Biochem Biophys Res Commun 340: 1259-1263, 2006.

37. van der Veen JN, Kruit JK, Havinga R, Baller JF, Chimini G, Lestavel S, Staels B, Groot PH, Groen AK and Kuipers F: Reduced cholesterol absorption upon PPARdelta activation coincides with decreased intestinal expression of NPC1L1. J Lipid Res 46: 526-534, 2005. 